\title{
Epigenetic Targeting in Cancer: Garden Variety Instructions?
}

\section{Mark T. Muller}

Department of Molecular Biology and Microbiology, College of Medicine, University of Central Florida, Orlando, FL 32826, USA

How DNA methylases target genomic sequences is a poorly understood, yet vital component in growth homeostasis in mammals. One mechanism of epigenetic targeting in animals has been shown to involve homology directed repair of double strand DNA breaks. This process leaves behind a methylation scar, which is a source of selective pressure that may promote carcinogenesis. Lessons from the plant reveal that small RNAs direct DNA methylation; however, in animals, RNA based methylation targeting is not understood. A recent report that RNA gene products regulate the DNA-damage response in animal cells, makes a strong imperative for a rigorous search for RNA based targeting mechanisms in humans.

Cytosine methylation is a post-replicative covalent modification of the genome associated with silencing of underlying genes [1]. The extent of cytosine methylation varies considerably among eukaryotes. In vertebrates, approximately $70-80 \%$ of cytosines in CpG dinucleotides are methylated. Invertebrates display a wide range of DNA methylation, from very limited methylation in Drosophilia melanogaster and Caenorhabditis elegans to a mosaic pattern of methylation in the sea urchin (Strongylocentrotus purpuratus).

Significant progress has been made in understanding DNA methylation in the model plant Arabidopsis thaliana. DNA methylation in plants differs somewhat from that of mammals. DNA methylation in mammals mainly occurs on the cytosine nucleotide, in a CpG site. In plants, the cytosine can be methylated at $\mathrm{CpG}, \mathrm{CpHpG}$, and $\mathrm{CpHpH}$ sites ( $\mathrm{H}$ represents any nucleotide but guanine). In both plants and animals, there are currently two types of DNA methylation: 1) a de novo type, using enzymes that create new methylation marks on the DNA; and 2) a maintenance type, acting on hemimethylated DNA and linked to DNA replication. The maintenance methylases in plants and mammals are MET1 and DNMT1, respectively, while de novo methylation events are mediated by DNMT3 in mammals, and DRM2 in Arabidopsis [2]. Current evidence suggests that for many (though not all) locations, RNA-directed DNA methylation (RdDM) is involved in targeting de novo action of DRM2. In RdDM, specific 24 nucleotide long RNA transcripts are produced from a genomic DNA template, and this RNA guides methylase action [3]. This sort of mechanism is thought to be important in cellular defense against endogenous RNA viruses and latent transposons, both of which are mutagenic to the host genome. By methylating their genomic locations, they are shut off and are no longer active in the cell. A relationship between DNA damage protection and RdDM is therefore well established in plants.

In mammalian somatic cells, methylation marks are heritable and stable. Moreover, their placement and removal is important in homeostatic growth control and in genome protection [4]. DNA methylation in somatic cells is layered at two levels. The first is stable methylation, also called imprinting, which is inherited in a sex-specific fashion and tends to be invariant between cells in different tissues [5]. The second level is somatic methylation, which tends to be variable between individuals and cell types. The second type of methylation affects all cells in the body, and impacts biological controls that direct growth versus no-growth decisions in the cell. Specifically in cancer, silencing by hypermethylation of a single growth suppressor pathway gene, can result in the loss of homeostatic controls. Another key feature in cancer is hypomethylation and genome instability, so the pendulum swings both ways. These findings define an important nexus linking epigenetics (DNA methylation) and growth control; however, we do not have a sound mechanistic understanding of this process since paradoxically, cancer cells display both genomic hyper- and hypomethylation epi-genotypes.

The process of DNA methylation is not sequence specific; therefore, an important over-riding issue remains unsolved. Specifically, how are genomic segments destined for methylation selected? In plants, $\mathrm{RdDM}$ is used to guide methylation enzymes to the site (see above); however, in higher eukaryotes the question is still open. We have been pursuing the idea that DNA damage may leave behind a scar, which is methylated DNA. We designed an experimental approach, to conclusively prove that this was the case. Using the system developed by Maria Jasin's lab [6], we analyzed expression patterns of GFP reporters derived from homologous recombination-DNA repair (HDR), after endogenous breakage by the homing endonuclease I-Sce1. Expression patterns of the GFP positive cells generated by HDR are bimodal, with two expression classes (in contrast, transfected wild type GFP expression is uniform in all cells). Roughly, half of these HDR cells are hypermethylated and under-express GFP, with the remainder being hypomethylated and over-expressed [7]. These results suggest that DNA methylation patterns are reprogrammed during HDR. This was confirmed by bisulfite sequence analysis that show the exact placement of 5 methyl cytosine in each repaired DNA $[7,8]$. The sequencing data further revealed the presence of distinctively different, but still related epi-alleles in the population of repaired cells. Moreover, the epigenetic changes map precisely to a region several hundred base pairs 3' of the DSB, (viz., the site of the end resection). More is now known about this mechanism of epigenetic reprogramming. For instance, key mediators (Gadd45a, Np95) are required, and the process is orchestrated by the maintenance methylase DNMT1 [9]. That the maintenance enzyme DNMT1 mediates this process was unexpected, since it is acting in a de novo capacity [7]. One explanation is that both HDR and DNMT1 are tightly coupled to $S$ phase.

Biological relevance of these findings, especially in light of the cancer growth defects are important; however, little is known about the frequency or penetrance of these events, as we age. On the other hand, even if HDR dependent epigenetic resets are rare events, their evolution may still hold physiological significance because growth positive phenotypes are selected, and retained. Since DNA damage is more or

*Corresponding author: Mark T. Muller, Department of Molecular Biology and Microbiology, College of Medicine, University of Central Florida, Orlando, FL 32826, USA, Tel: 407-882-2268; E-mail: Mark.Muller@ucf.edu

Received September 25, 2012; Accepted September 25, 2012; Published September 30, 2012

Citation: Muller MT (2012) Epigenetic Targeting in Cancer: Garden Variety Instructions? J Plant Pathol Microb 3:e103. doi:10.4172/2157-7471.1000e103

Copyright: (C) 2012 Muller MT, et al. This is an open-access article distributed under the terms of the Creative Commons Attribution License, which permits unrestricted use, distribution, and reproduction in any medium, provided the original author and source are credited. 
less randomized across the genome, the entire process is stochastic and resulting growth phenotype depends largely on the genomic context of the epigenetic revision. Epigenetic revisions would be tolerated if the repaired segments do not impact critical growth regulatory genes. On the other hand, a tumor suppressor gene may occasionally be silenced which would lead to a cell with a growth advantage. The process then defines a potentially powerful evolutionary force, since rare alterations in the epigenetic landscape can lead to amplification of a sub-population of growth promoted cells. The recent and surprising evidence from the ENCODE Project that a large fraction (76\%) of the human genome is transcribed into RNA [10], suggests that repair based methylation revisions are more likely to target genes that produce RNA as the gene product (as opposed to protein encoding genes). In fact, RNA based regulation and targeting in chromatin is an important topic, under intense study $[11,12]$. Further support for RdDM-like mechanisms in somatic cell HDR comes from recent work showing that the DNA damage response is directed by non-coding RNA, working at sites of DNA damage in human and zebrafish models [13]. A connection between methylation revisions in HDR through an RdDM type of mechanism seems logical and likely.

Future research will improve the veracity of the model. Other questions remain open. In animal cells, for example, non-homologous end joining (NHEJ) represents a second major DS break repair pathway, which operates outside of S/G2 phases. NHEJ is highly error prone (in contrast to HDR), since it involves simple trimming and religation of free DNA ends. It would be interesting to search for epigenetic revisions, following NHEJ, that leave behind a historical record in the form of a methylation scar. Finally, epigenetic revisions may have predictive value in epigenomic profiling of tumors. Specifically, in cancers with silenced tumor suppressor genes, one could reconstruct the precise location of DS DNA breaks, based on bisulfite sequence profiles in tumors from patients. Bioinformatic rendering of these data (ClustalW), in fact, reveals the relatedness of epi-alleles and should help define an epigenetic evolutionary process of carcinogenesis [7].

\section{References}

1. Bird A (2002) DNA methylation patterns and epigenetic memory. Genes Dev 16: 6-21.

2. Feng S, Jacobsen SE, Reik W (2010) Epigenetic reprogramming in plant and animal development. Science 29: 622-627.

3. Aufsatz W, Mette MF, van der Winden J, Matzke AJ, Matzke M (2002) RNA directed DNA methylation in Arabidopsis. Proc Natl Acad Sci USA 99: 16499 16506.

4. You JS, Jones PA (2012) Cancer Genetics and epigenetics: Two sides of the same coin. Cancer Cell 22: 9-20.

5. Barlow DP (2011) Genomic imprinting: a mammalian epigenetic discovery model. Annu Rev Genet 45: 379-403.

6. Weinstock DM, Brunet E, Jasin M (2008) Induction of chromosoma translocations in mouse and human cells using site-specific endonucleases. $J$ Natl Cancer Inst Monogr 39: 20-24.

7. Cuozzo C, Angrisano T, Porcellini A, Morano A, Messina S, et al.(2007) Homology Directed Repair and DNA Methylation. PLoS Genetics 3: 1144-1162.

8. Lee GE, Kim JH, Taylor M, Muller MT (2010) DNA Methyltransferase 1-associated Protein (DMAP1) Is a co-repressor that stimulates DNA methylation globally and locally at sites of double strand break repair. $\mathrm{J}$ Bio Chem 285: 37360-37340

9. Lee B, Morano A, Porcellini A, Muller MT (2012) GADD45a inhibition of DNMT1 dependent DNA methylation during homology directed DNA repair. Nucleic Acids Res 40: 2481-2493.

10. Pennisi E (2012) Genomics.ENCODE Project writes eulogy for junk DNA Science 337: 1159-1161.

11. Rinn JL, Chang HY (2012) Genome regulation by long noncoding RNAs. Annu Rev Biochem 81: 145-166.

12. Denis H, Ndlovu MN, Fuks F (2011) Regulation of mammalian DNA methyltransferases: a route to new mechanisms. EMBO Rep 12: 647-656.

13. Francia S, Michelini F, Saxena A, Tang D, de Hoon M, et al. (2012) Site-specific DICER and DROSHA RNA products control the DNA-damage response. Nature 488: 231-235. 